\title{
LOCALIZING PRIME IDEMPOTENT KERNEL FUNCTORS
}

\author{
S. K. $\operatorname{SIM}^{1}$
}

ABSTRACT. In this note, we call a prime idempotent kernel functor a localizing prime if it has the so-called property ( $T$ ) of Goldman. We generalize a theorem of Heinicke to characterize localizing prime idempotent kernel functors and present an example of a prime idempotent kernel functor on Mod- $R$, the category of unitary right $R$-modules, which is not a localizing prime, even though $R$ is a right artinian ring.

We shall in most cases follow the terminology in [1]. All rings are assumed to have a unit element and all modules are unitary right modules. Let $\sigma$ be an idempotent kernel functor on $\operatorname{Mod}-R$, i.e., a left exact subfunctor of the identity functor on Mod- $R$ such that $\sigma(M / \sigma(M))=0$ for all $M \in \operatorname{Mod}-R$. We denote the module of quotients of $M$ with respect to $\sigma$ by $M_{\sigma^{\circ}}$. To each $R$-module $S$, there is an associated idempotent kernel functor $\tau_{S}$ given by the formula $\tau_{S}(M)=\{m \in M \mid f(m)=0$ for all $f: M \rightarrow E\}$, where $E$ is the injective hull of $S$. In case $S=R / I$ for some two-sided ideal $I$ of $R$, we write $\mu_{I}$ for $\tau_{S}$, and $M_{I}$ for the module of quotients of $M$ with respect to $\mu_{I}$.

An idempotent kernel functor $\sigma$ on $\operatorname{Mod}-R$ is called a prime if $\sigma=\tau_{S}$, where $S$ is a supporting module for $\sigma$, i.e., $S$ is $\sigma$-torsion free and $S / S^{\prime}$ is $\sigma$-torsion for each nonzero submodule $S^{\prime}$ of $S$. A prime idempotent kernel functor $\sigma$ on Mod- $R$ is called a localizing prime if every $R \sigma^{\text {-module is }}$ $\sigma$-torsion free as $R$-module. In other words, an idempotent kernel functor $\sigma$ on Mod- $R$ is a localizing prime if and only if $\sigma$ is a prime with Goldman's property (T) (see [1, The ore $\mathrm{m}$ 4.3]).

In [1] Goldman has shown that if $A$ is a commutative ring, then an idempotent kernel functor $\sigma$ on $\operatorname{Mod}-A$ is a prime if and only if $\sigma=\mu_{P}$ for some

Received by the editors June 28, 1973.

AMS (MOS) subject classifications (1970). Primary 16 A08.

Key words and phrases. Idempotent kernel functor, prime idempotent kernel functor, property (T), localizing prime idempotent kernel functor, module of quotients, ring of quotients, supporting module, irreducible module, right artinian ring, right noetherian ring, socle.

1 This paper is a portion of the author's doctoral dissertation written at the University of Westem Ontario under the direction of Dr. A. G. Heinicke. 
prime ideal $P$ of $A$. Thus every prime idempotent kernel functor on Mod- $A$ is a localizing prime. However, for a noncommutative ring, this is not always true.

From [3, Corollary 3.10], we know that if $R$ is a right noetherian ring, then for each prime ideal $P$ of $R, \mu_{P}$ is a prime. In [2, Theorem 4.3] Heinicke has shown that $\mu_{P}$ is a localizing prime if and only if all irreducible $R_{P}$-modules are isomorphic and the socle of the $R_{P}$-module $(R / P)_{P}$ is nonzero. We shall show that this result can be generalized to characterize localizing prime idempotent kernel functors on Mod- $R$ without assuming any chain condition on $R$.

Lemma. Let $\sigma$ be an idempotent kernel functor on Mod- $R$ with the property that every $R_{\sigma}$-module is $\sigma$-torsion free as an $R$-module. Then an $R_{\sigma}{ }^{-m o d u l e}$ is irreducible if and only if it is a supporting module for $\sigma$ when regarded as an $R$-module.

The proof of this lemma is straightforward and so will be omitted.

Theorem. Let $\sigma$ be an idempotent kernel functor on Mod-R. Then the following are equivalent:

(1) $\sigma$ is a localizing prime.

(2) All irreducible $R_{\sigma}$-modules are isomorphic and, for each $R$-module $M$ such that $\sigma=\tau_{M}$, the $R_{\sigma}$-module $M_{\sigma}$ bas nonzero socle.

(3) All irreducible $R_{\sigma}$-modules are isomorpbic, and there exists an

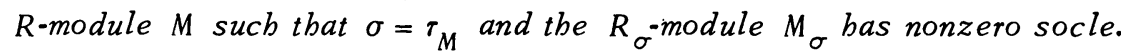

(4) All irreducible $R_{\sigma}$-modules are isomorphic and $\sigma$-torsion free as $R \cdot$ modules.

Proof. $(1) \Rightarrow(2)$. Since $\sigma$ is a prime idempotent kernel functor on $\operatorname{Mod}-R$, all $\sigma$-injective supporting modules are isomorphic as $R$-modules by [1, Theorem 6.4]. It follows from the Lemma that all irreducible $R_{\sigma}$-modules are isomorphic as $R_{\sigma}$-modules.

Let $M$ be an $R$-module such that $\sigma=\tau_{M}$. Then $M$ contains a supporting module, say $U$, for $\sigma$. Since $U_{\sigma}$ is an irreducible $R_{\sigma}$-submodule of $M_{\sigma}$, the $R_{\sigma}$-module $M_{\sigma}$ has nonzero socle.

$(2) \Rightarrow(3) \Rightarrow$ (4) is clear.

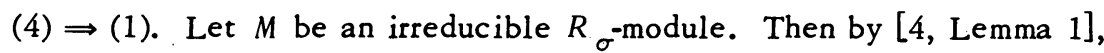
the $R_{\sigma}$-injective hull (which is also the $R$-injective hull) of $M$ is a cogenerator of Mod- $R_{\sigma}$ which is $\sigma$-torsion free as an $R$-module. Hence every

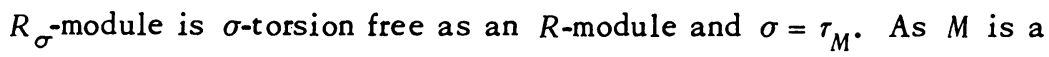


supporting module for $\sigma$ by the Lemma, $\sigma$ is a prime idempotent kernel functor.

Remark. If $\sigma$ is a localizing prime idempotent kernel functor on Mod- $R$, then all irreducible $R_{\sigma}$-modules are isomorphic, and so the Jacobson radical of $R_{\sigma}$ is the unique maximal two-sided ideal.

Finally, we present an example show ing that a prime idempotent kernel functor on Mod- $R$ need not be a localizing prime, even if $R$ is right artinian.

Example. Let $R$ be the subring of the complete $3 \times 3$ matrix ring over a field $F$ consisting of elements of the form

Then

$$
\left[\begin{array}{lll}
a & b & c \\
0 & d & e \\
0 & 0 & a
\end{array}\right]
$$

and

$$
P=\left\{\left[\begin{array}{lll}
0 & b & c \\
0 & d & e \\
0 & 0 & 0
\end{array}\right] \mid b, c, d, e \in F\right\}
$$

$$
K=\left\{\left[\begin{array}{lll}
a & b & c \\
0 & 0 & e \\
0 & 0 & a
\end{array}\right] \mid a, b, c, e \in F\right\}
$$

are two-sided ideals which are also maximal right ideals of $R$.

Consider the prime idempotent kernel functor $\sigma=\mu_{P}$. One can show that $K$ is the smallest right ideal of $R$ for which $R / K$ is $\sigma$-torsion and then deduce that $\sigma(R)=0$ and $R_{\sigma}=\operatorname{Hom}_{R}(K, R)$. Now, if $\sigma$ were a localizing prime, then $K$ would have to be a projective $R$-module, which is not the case.

\section{REFERENCES}

1. O. Goldman, Rings and modules of quotients, J. Algebra 13 (1969), 10-47. MR 39 \#6914.

2. A. G. Heinicke, On the ring of quotients at a prime ideal of a right Noetherian ring, Canad. J. Math. 24 (1972), 703-712. MR 45 \#8681.

3. J. Lambek and G. Michler, The torsion theory at a prime ideal of a right Noetherian ring, J. Algebra 25 (1973), 364-389.

4. B. L. Osofsky, A generalization of quasi-Frobenius rings, J. Algebra 4 (1966), 373-387. MR $34 \# 4305$.

DEPARTMENT OF MATHEMATICS, UNIVERSITY OF WESTERN ONTARIO, LONDON, ONTARIO, CANADA

Current address: Departamento de Matemáticas, Facultad de Ciencias, Universidad Central de Venezuela, Caracas, Venezuela 